\title{
Medicaid claims data to supplement Zika-related birth defects case identification
}

\author{
Carrie W. Mills*, Tenzin Tseyang, Katharine McVeigh and George Askew \\ NYC Department of Health and Mental Hygiene, Long Island City, NY, USA
}

\section{Objective}

To assess the use of Medicaid claims data to conduct surveillance for cases of Zika-related birth defects identified after birth among infants born in New York City (NYC).

\section{Introduction}

As a part of the Zika Birth Defects Surveillance, a national effort coordinated by the Centers for Disease Control and Prevention (CDC), $\mathrm{NYC}$ is conducting enhanced surveillance of all births with defects included in the congenital Zika syndrome (CZS) phenotype among infants born in NYC beginning in 2016. The intent of the project is to provide background on the prevalence of these conditions, regardless of cause. The surveillance project builds on the New York State (NYS) Congenital Malformations Registry, a passive, mandatory reporting system that relies on reporting from hospitals and providers. For the Surveillance project, potential cases of Zika-related birth defects (ZBD) are identified by hospital and administrative data of birth records with one or more of the International Classification of Diseases, 10th Revision (ICD-10) diagnostic codes associated with CZS. ${ }^{1}$ The list of included diagnostic codes was specified by the NYS registry following guidance established by CDC. Full medical record chart abstraction of the birth hospital visit of potential cases is then conducted applying further inclusion guidelines to identify ZBD cases.

Recent reports of late presentation of birth defects consistent with CZS suggest that some cases are being missed due to identification and diagnosis of the condition after birth. ${ }^{2}$ As one component of a broader strategy to obtain a more accurate surveillance count, we seek to identify potential ZBD cases first diagnosed in the 6-month postpartum period using Medicaid claims data.

\section{Methods}

We will obtain Medicaid records for all infants born in NYC in 2016 from Jan 1, 2016 through June 30, 2017 using Salient, a data mining system of Medicaid data (Salient Interactive Miner, Version 5.70.079). The 85 ICD-10 diagnostic codes currently being used to identify potential ZBD cases will be applied to birth records and all outpatient and inpatient visits to a medical provider for the 6-month period after birth. All visits containing one or more of the codes from either primary or secondary diagnosis will be identified. A unique list of infants receiving one or more included diagnoses within the 6-month postpartum period will be obtained and cross-referenced with the current case list using a matching algorithm based on child's name, date of birth, and other identifying variables.

\section{Results}

\section{Preliminary results}

Surveillance measures to-date have identified 380 cases of infants born in NYC in 2016 with birth defects that could be due to Zika virus; it is anticipated that a majority have Medicaid. (In 2015, 59\% of all births in NYC were to mothers with Medicaid.)

Analysis will determine (a) the extent of overlap of cases identified from surveillance activities and Medicaid claims data, and (b) the extent of ZBD potential cases missing from surveillance but found with Medicaid data of in- and out-patient visits. Descriptive statistics will include age and class of earliest diagnosis of infants. Those identified by Medicaid analysis will be considered potential ZBD cases pending full abstraction of record.

Full results pending.

\section{Conclusions}

If results indicate missed potential ZBD cases, medical chart abstraction of such cases will be warranted. Further, as CZS is a relatively new syndrome, findings may provide support in the determination of accurate follow-up time for future surveillance projects. $^{3}$

Full conclusion pending.

\section{Keywords}

birth defects; Zika; Medicaid; Surveillance

\section{Acknowledgments}

Congenital Malformations Registry, New York State Department of Health

NYC Zika Pregnancy Registry, NYC Department of Health and Mental Hygiene

\section{References}

1. Moore CA, Staples JE, Dobyns WB, et al. Characterizing the Pattern of Anomalies in Congenital Zika Syndrome for Pediatric Clinicians. JAMA Pediatrics. 2017;171(3):288-295. doi:10.1001/ jamapediatrics.2016.3982.

2. Cragan JD, Mai CT, Petersen EE, et al. Baseline Prevalence of Birth Defects Associated with Congenital Zika Virus Infection Massachusetts, North Carolina, and Atlanta, Georgia, 2013-2014. MMWR Morb Mortal Wkly Rep. 2017;66:219-222. DOI: http://dx.doi. org/10.15585/mmwr.mm6608a4.

3. Shapiro-Mendoza CK, Rice ME, Galang RR, et al. Pregnancy Outcomes After Maternal Zika Virus Infection During Pregnancy - U.S. Territories, January 1, 2016-April 25, 2017. MMWR Morb Mortal Wkly Rep. 2017;66:615-621. DOI: http://dx.doi.org/10.15585/ mmwr.mm6623e1.

\section{*Carrie W. Mills}

E-mail: cmills@health.nyc.gov 